\title{
AN ALYS IS
}

ing extension to human systems in clinical settings?

Probably foremost among them is whether one can, or should, use adult tissue as a source of SCs in autologous graft paradigms. The alternative to this approach, in transplantation-based strategies, is the use of stable SC lines as "universal donor cells." The latter has the appeal of being an "off-the shelf" reagent, prepared and/or additionally engineered under good manufacturing practices readily available in limitless quantities for the acute phases of an injury or disease. Its downside is the possibility of immune incompatability. An additional concern is whether the starting material for such lines might initially need to come (albeit just initially) from a developmental stage (embryo, fetus, adult) that empirically proves optimal for yielding potent SCs. (Prenatal sourceswhich may prove biologically optimal-are colored by an ethical dimension not typically borne by adult tissues.)

While grafting a patient's own SCs might circumvent these problems, the prospective isolation, expansion, characterization, and directed differentiation of cellular reagents for each new patient-with its attendant costs in time, resources, manpower, and potential inter-preparation variability-represents a considerable challenge. This also presumes that we know how to translate observations like those of Clarke et al. for practical use, which, in turn, presupposes a knowledge of the signals involved and an ability to provide them controllably-far from realized.

Is it necessary to expose adult SC s to the primitive blastula environment for expression of a more generalized lineage potential? An embryonic milieu may reactivate a subset of options (e.g., kidney, heart), but not others (e.g., certain mesodermalderived cells $\left.s^{1}\right)$, whereas exposure to an adult milieu may be necessary to promote these other repertoires (e.g., skeletal muscle, blood)2,3. One must also create safeguards such that cells with theoretical "totipotency" do not give rise to inappropriate cells (e.g., muscle in brain), transform to teratocarcinomas, or create autonomous organs within the larger organ (e.g., neural tubes within the heart). Although pre-differentiation ex vivo might preclude this, the comfort of invariant commitment must be weighed against the loss of plasticity and multipotency, wherein the environment directs SC s toward needed phenotypes with potential reconstitution of degenerated regions with multiple cell types $^{8}$. (Interestingly, despite the extensive pluripotency suggested for neural SCs by recent papers ${ }^{1,2}$, undesired cells have never been observed in intracranial transplant paradigms ${ }^{8-13}$.) An additional consideration with the autograft strategy is more fundamental than technical: though possibly useful for trauma-based deficits, it would probably not be optimal for genetically based diseases (i.e., reimplanting cells al ready harboring a defect or predisposition).

Human versions of organ-specific ${ }^{6,9-15}$ and embryonic SC s s-18 are available for laboratory experimentation. They emulate many of the characteristics of their rodent counterparts, suggesting great promise for their ultimate use in clinical situations. Each of the questions posed above, therefore, will be carried forward to the conference rooms of real hospitals: totipotent vs. organ-specific cells; embryonic- vs. fetal- vs. adult-derived cells.

A paper, such as that by Clarke et al., suggesting that adult organ-specific cells may fulfill the role of ES cells, simply makes the debate-particularly the ethical dimensions-all the more intense and interesting. At present, the idea that adult SC s are equivalent to, and may replace, embryonic SCs in basic and therapeutic investigations is not solidly founded. H owever, the recent spate of

studies suggesting unexpected degrees of plasticity are certain to stimulate experiments that, three years ago, may have been unimaginable.

1. Clarke, D.L., et al. Science 288, 1660-1663 (2000)

2. Bjornson, C.R., et al., Science 283, 534-537 (1999).

3. Gussoni, E., et al., Nature 401, 390-394 (1999).

4. Jackson, K.A., Mi, T. \& Goodell, M.A., Proc. Natl. Acad. Sci. USA 96, 14482-14486 (1999).

5. Kopen, G.C., Prockop, D.J \& P Pinney, D.G., Proc. Natl. Acad. Sci. USA 96, 10711-10716 (1999).

6. Pittinger, M.F Science 284, 143-147 (1999).

7. Vijayakumar, K.R. et al. Nat. Med. 6, 278-282 (2000).

8. Yandava, B.D., Billinghurst, L.L. \& Snyder, E.Y. Proc. Natl. Acad. Sci. USA, 96, 7029-7034 (1999).

9. Svendsen, C.N. et al. Exp. Neurol. 148, 135-146 (1997).

10. Vescovi, A.L. et al. Exp. Neurol. 156, 71-83 (1999).

11. Fricker, R.A. et al. J. Neurosci. 19, 5990-6005 (1999).

12. Brüstle, O. et al. Nat. Biotechnol. 11, 1040-1049 (1998).

13. Flax, J.D. et al. Nat. Biotechnol. 16, 1033-1039 (1998).

14. Roy, N.S. et al. Nat. Med. 6, 271-277 (2000)

15. Villa, A. et al. Exp. Neurol. 161, 67-84 (2000).

16. Thompson, J. et al. Science 282, 1145 (1998).

17. Shamblott, M.J. . et al. Proc. Natl. Acad. Sci. USA 95 13726-13731 (1998).

18. Reubnoff, B.E. et al. Nat. Biotechnol. 18, 399-404 (2000).

\section{Biological software for materials engineering}

\section{Roberto Cingolani}

The cross-fertilization of biology, chemistry, and materials science is opening up tremendous opportunities for innovation in previously unrelated disciplines such as electronics and information processing. The extraordinary recognition capability of biomolecules has suggested their use as a means for direct recognition and binding of inorganic materials-in other words acting as a sort of natural software for programming the formation of matter. A recent paper in Nature by Whaley et al. ${ }^{1}$, which describes an approach for generating peptides suitable for use in semiconductors and metal binding, is the latest of a series of papers $^{2-8}$ that demonstrate the potential of these biological approaches in materials engineering.

Researchers working in fields as disparate as molecular electronics and the biology of bone formation are becoming

Roberto Cingolani is professor, IN FM Research Unit, Department of Innovation Engineering, University of Lecce, Via Arnesano 73100 Lecce, Italy (roberto.cingolani@unile.it). increasingly aware of the use of self-assembling biomolecules as tools for studying and controlling the formation of complex structures, both organic and inorganic (metals and semiconductors). The main idea is to exploit the highly specific binding properties characteristic of naturally formed organisms to drive the formation of hybrid inorganic structures containing semiconductors or metals.

By way of example, Whaley et al. have screened a combinatorial phage library of millions of peptides with unknown binding properties against inorganic molecules and succeeded in selecting specific peptide sequences that can distinguish different crystallographic planes of the most important semiconductors (gallium arsenide and silicon). These peptides could therefore be used to control the positioning and the assembly of materials at the nanoscale.

A somewhat similar approach has been pursued by Brown and coworkers ${ }^{2}$, but in a totally different context. In this case, the authors were interested in investigating peptide-driven formation of gold crystals, as a prototype mechanism for the formation of natural solids like bones and teeth in 
the human body. They have developed a genetic system in the bacterium Escherichia coli to control the shape, size, and orientation of gold crystals, showing that the peptides govern gold crystallization through a catalytic reaction induced by an acid mechanism.

Several other groups are highlighting the increasing importance of self-assembling biomolecules for electronic hybrid systems. Braun and colleagues ${ }^{3}$ have hybridized DNA molecules with surfacebound oligonucleotides as a means of stretching the nucleic acid backbone in between gold electrodes of a metallic network. In principle, this is the first example of a self-organized hybrid electronic network where the biomolecules act as intelligent wires, forming the circuits, whereas the metallic part is used merely as a macroscopic interface to the real world. Since the DNA used for the network formation showed no conductivity, these authors deposited silver clusters on the DNA strands to yield a conductive network. Because of the excellent mechanical properties of the strand (the wires were $12 \mu \mathrm{m}$ long and $100 \mathrm{~nm}$ thick), the biomolecule served not only as a vectorial driver, but also as a mechanical support for the deposition of the silver clusters.

This work highlights increasing interest in the mechanical properties of DNA and its electrical conduction. Several groups are currently investigating DNA's mechanical properties, including its elastic force constant, its capability to be elongated more than 70 times its resting length, and the friction coefficient of a single molecule ${ }^{4}$. Elsewhere, anisotropic conductivity along the DNA strand has been convincingly shown by a Japanese group ${ }^{5}$, who measured the electrical transport across an interdigitated metallic contact filled by DNA molecules oriented in an organic film. In another study, Porath and colleagues ${ }^{6}$ have electrostatically trapped a single $10.4 \mathrm{~nm}$ long DNA molecule in a free-standing metallic "nanogate," demonstrating that the current voltage of the system resembled diodelike behavior. Fink and Schönenberger ${ }^{7}$ have also measured the charge transport through single "ropes" (600 nm long) of DNA by low-energy electron point source microscopy. A resistance of the order of 2-3 $\Omega$ was measured across the rope, showing no rectification (i.e., deviation from O hm's Law) in the current-voltage characteristics. Elsewhere, Rinaldi and colleagues ${ }^{8}$ have measured a large $\pi-\pi$ stack conductivity, with clear rectification and hysteresis (dependence of current in the device according to whether the voltage is swept upwards or downwards) in the current voltage characteristics in nanogates interconnected by self-assembled guanosine oligonucleotides, with a peculiar order/disorder transition of the molecular film in the gates. This suggests that a solid-state film consisting of self-organized DNA behaves in a way similar to a semi-conductor.

The above experiments (and many others) exemplify the increasing overlap between biology, chemistry, and physics. The

\section{Quantum dots}

Considerable interest is also focusing on artificial atoms, termed quantum dots, that can be fabricated by self-organized epitaxy (growth of an ultrapure crystalline layer) of III-V semiconductors (e.g. gallium arsenide). Work in my group has demonstrated that these quantum dots exhibit full quantization of electonic states, descretization of energy levels, and Zeeman splitting (i.e., splitting of energy levels according to the application of an external magnetic

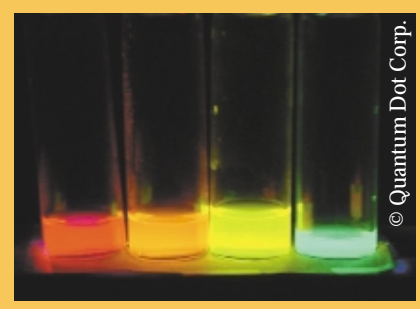
field), just like natural atoms ${ }^{9}$. Artificial molecules consisting of coupled quantum dots have also been created by exploiting the precision of electron beam lithographies ${ }^{10}$. Such quantum dots exhibit the properties of natural atoms and molecules scaled on a more macroscopic level, thus opening up several interesting fundamental studies. On the other hand, the possibility of engineering energy levels and wave functions through the choice of material and control of quantum dot size has important applications in electron memories, new solid state lasers, quantum computing, and ultimately for nanoscale devices that chould be used as sensors and templates for the self-assembly of biomolecules. ultimate reason for this is that some of the basic mechanisms controlling the formation of complex natural systems and their operation (e.g., cognition in the brain) are becoming very appealing for artificial technologies like information processing and electronics, where conventional semiconductor physics is coming closer to its natural limit.

What is absolutely certain is that new technologies are needed to study processes at the molecular and single-molecule level. In this respect, the availability of instruments with atomic resolution, such as scanning tunneling microscopy and atomic force microscopy, will beincreasingly important in enhancing our ability to observe and manipulate nano-objects. In addition to new visualization technologies, we will need new synthetic methods to complement high-resolution lithographies for nanometer-scale fabrication.

Biological molecules offer considerable advantages over inorganic matter in terms of recognition and self-assembly for materials engineering. We are clearly only just beginning to glimpse the potential of biology as software for programming the formation of complex systems.

1. Whaley, S.R. et al. Nature 405, 665-668 (2000).

2. Brown, S., Sarikaya, M. \& J ohnson, E. J. Mol. Biol. in press $(2000)$

3. Braun, E., Eichen, Y., Sivan, U. \& Ben-Yoseph, G. Nature 391, 775-778 (1998).

4. Wirts, D. Phys. Rev. Lett. 75, 2436-2439 (1995).

5. Okahata, Y., Kobayashi, T., Tanaka, K. \& Shimomura, M. J. Am. Chem. Soc. 120, 6165-6166 (1998).

6. Porath, D., Bezryadin, A., DeVries, S. \& Dekker, S. Nature 403, 635-638 (2000).

7. Fink, H.W. \& Schönenberger, C. Nature 398, 407-410 (1999).

8. http://arxiv.org. Rinaldi, R. et al. cond-mat/0006402

9. Cingolani, R. et al. Phys. Rev. Lett. 83, 4832-4835 (1999).

10. Oosterkamp, T.H. et al. Nature 395, 873-876 (1998). 